\title{
Análisis de las relaciones entre la legitimidad organizativa, sus fuentes y dimensiones*
}

\author{
Cruz-Suárez, Ana** \\ Díez-Martín, Francisco*** \\ Blanco-González, Alicia**** \\ Prado-Román, Camilo****
}

\section{Resumen}

En esta investigación se analizan dos relaciones planteadas desde el campo de la Teoría Institucional: 1) las diferencias que se producen entre la legitimidad organizativa y sus dimensiones, y 2) la relación entre la legitimidad organizativa y sus fuentes. El estudio de estas relaciones se realiza sobre el grado de legitimidad alcanzado por el Espacio Europeo de Educación Superior en las universidades públicas de la Comunidad Autónoma de Madrid (España). Para ello se ha utilizado el análisis de regresión múltiple sobre 1544 encuestas. Los resultados han indicado que las dimensiones pragmática, moral y cognitiva, influyen positivamente sobre la legitimidad. Sin embargo, el grado de influencia es dispar. A su vez, las evaluaciones de las fuentes de legitimidad también han mostrado divergencias entre sí. Las conclusiones de este estudio suponen un avance en la literatura sobre la Teoría Institucional, pues confirman empíricamente relaciones planteadas de forma teórica.

Palabras clave: Legitimidad organizativa, dimensiones de legitimidad, fuentes de legitimidad, espacio europeo de educación.

Recibido: 15-12-12. Aceptado: 15-10-13

* Esta investigación ha sido financiada por la Dirección General de Universidades e Investigación de la Consejería de Educación de la Comunidad Autónoma de Madrid, y por la Universidad Rey Juan Carlos, Ref: URJC-CM-2010-CSH-5186. Se expresa especial agradecimiento al profesor Emilio Díez de Castro por sus recomendaciones y sugerencias, indispensables para el desarrollo de esta investigación.

** Doctora en Dirección Estratégica, Profesora e investigadora del Departamento de Economía de la Empresade la Universidad Rey Juan Carlos, e-mail: ana.cruz@urjc.es

*** Doctor en Dirección Estratégica. Universidad de Sevilla. Profesor e investigador del Departamento de Economía de la Empresa de la Universidad Rey Juan Carlos, e-mail: francisco.diez @urjc.es

**** Doctora en Marketing, Profesora e investigadora del Departamento de Economía de la Empresa de la Universidad Rey Juan Carlos, e-mail: alicia.blanco@urjc.es

***** Doctor en Economía Financiera, Profesor e investigador del Departamento de Economía de la Empresa de la Universidad Rey Juan Carlos, e-mail: camilo.prado.roman@urjc.es 


\title{
Analysis of Relations between Organizational Legitimacy, its Sources and Dimensions
}

\begin{abstract}
This research analyzed two relationships proposed in the field of institutional theory: 1) the differences produced between organizational legitimacy and its dimensions, and 2) the relation between organizational legitimacy and its sources. Study of these relationships is carried out based on the degree of legitimacy reached by the European Space for Higher Education in public universities of the Autonomous Community of Madrid (Spain). To accomplish this, multiple regression analysis was used on 1544 surveys. Results indicated that the pragmatic, moral and cognitive dimensions have a positive influence on legitimacy. Nevertheless, the degree of influence is disparate, and evaluations of the sources of legitimacy have also demonstrated divergences among themselves. Conclusions suppose an advance in the literature about Institutional Theory, because they empirically confirm the relationships proposed in a theoretical form.
\end{abstract}

Key words: Organizational legitimacy, dimensions of legitimacy, sources of legitimacy, European Space for Education.

\section{Introducción}

La legitimidad es un concepto fundamental en la Teoría Institucional (Senés y Llamas, 2004; Cruz-Suárez et al., 2014). Su estudio se remonta a los trabajos de Weber (1946) quién introdujo el tema en el campo de la sociología y la teoría de las organizaciones (Ruef y Scott, 1998). Este autor la considera como el resultado de la conformidad con las normas socialmente establecidas y las leyes gubernamentales. Para Scott (1995) consiste en un estado que refleja la alineación cultural, el apoyo normativo o la consonancia con las reglas y leyes relevantes, es "la percepción generalizada o asunción de que las actividades de una entidad son deseables, correctas o apropiadas dentro de algún sistema socialmente construido de normas, valores, creencias y definiciones" (Suchman, 1995: 574).

Algunos autores consideran que representa un factor clave de éxito para la supervivencia de las organizaciones (Me- yer y Rowan, 1977; Zucker, 1987; Zimmerman y Zeitz, 2002). Su importancia radica en que la aceptación y deseabilidad de las actividades de una organización, posibilitará que ésta acceda a los recursos necesarios para sobrevivir y crecer (Baum y Oliver, 1991). Brown (1998: 35) sugiere que tener legitimidad es "una condición necesaria (de las organizaciones) para conseguir el fácil acceso a los recursos, a los mercados y a la supervivencia a largo plazo".

Investigaciones recientes han analizado diferentes aspectos de la legitimidad en el ámbito de las organizaciones: a) se han identificado acciones estratégicas que mejoran su legitimidad (Suchman, 1995; Zimmerman y Zeitz, 2002); b) también se ha teorizado sobre las distintas dimensiones que la conforman (Meyer y Rowan,1977; DiMaggio y Powell, 1983; Aldrich y Fiol, 1994; Scott, 1995; Suchman, 1995; Greenwood et al., 2002; Rutherfor y Buller, 2007); c) otros 
estudios han analizado la relación entre las estrategias de legitimación y la supervivencia (Bansal y Clelland, 2004; Barreto y Baden-Fuller, 2006; Tornikoski y Newbell 2007; Low y Johnston, 2008), d) así como la relación entre las dimensiones de la legitimidad y la supervivencia (Deephouse, 1996; Ruef y Scott, 1998; Li et al., 2007; Díez-Martín et al., 2013).

Si bien estos estudios han analizado distintos aspectos que envuelven el concepto de legitimidad, aún persisten algunos vacíos que deben aclararse. En primer lugar, existe la necesidad por conocer las diferencias que se producen entre la legitimidad organizativa y sus dimensiones (Suchman, 1995). Los investigadores en este campo consideran que la legitimidad es un concepto multidimensional (Thomas, 2005), pero no han podido determinar la fuerza de cada dimensión. Este conocimiento permitiría a las organizaciones dirigir sus esfuerzos hacia aquellas dimensiones que aumentasen en mayor grado la legitimidad de la organización.

En segundo lugar, algunos autores también sugieren la necesidad de aclarar la relación entre los grupos que otorgan legitimidad (fuentes de legitimidad), y las dimensiones que la componen (Zimmerman y Zeitz, 2002). En esta línea de pensamiento, Díez-Martín et al. (2010a) se preguntan ¿qué fuente ejerce un peso mayor sobre la legitimidad de una organización? Igualmente, este conocimiento permitiría a las empresas desarrollar acciones sobre aquellos grupos que otorgan mayor legitimidad a las organizaciones.

De esta forma, el propósito central de esta investigación consiste en 1) anali- zar las diferencias que se producen entre la legitimidad organizativa y sus dimensiones y, 2) analizar las relaciones entre la legitimidad organizativa y sus fuentes.

Para alcanzar tales objetivos, el estudio se centra en el Espacio Europeo de Educación Superior (EEES), al considerar que representa un escenario pertinente para estudiar las relaciones a explorar, al tratarse de un proceso institucional que puede ser sujeto de legitimación (Johnson, 2004). Así, se analiza la legitimidad del EEES por medio de sus grupos de interés (Ruef y Scott, 1998), formados por la comunidad universitaria. En este caso se estudia a la comunidad universitaria de las seis universidades públicas de la Comunidad Autónoma de Madrid (CAM).

La muestra utilizada en la investigación está compuesta por 761 profesores y 783 alumnos de grado de las seis universidades. El número de alumnos y profesores encuestados se encuentra en proporción a los totales de cada universidad. El error muestral para el conjunto de alumnos, con un nivel de significatividad del $95 \%$, ha sido de 3,460 , similar al de los profesores $(3,469)$. Puesto que la investigación se realiza sobre dos grupos poblaciones, se considera necesario comprobar la existencia de diferencias en las varianzas de las opiniones generadas por ambos: para ello se ha llevado a cabo un análisis de las varianzas (ANOVA).

Para analizar las diferencias que se producen entre la legitimidad organizativa y sus dimensiones se ha utilizado el análisis de regresión múltiple, siguiendo el método de introducción por pasos. En este caso, la variable dependiente sería la legitimidad del EEES y las variables independientes sus dimensiones. Para las 
Análisis de las relaciones entre la legitimidad organizativa, sus fuentes y dimensiones Cruz-Suárez, A.; Díez-Martín, F.; Blanco-González, A. y Prado-Román, C.

dimensiones se utiliza la clasificación propuesta por Suchman (1995): pragmática, moral y cognitiva. Por otro lado, el estudio de la relación entre la legitimidad organizativa y sus fuentes se llevó a cabo analizando las diferencias mostradas por profesores y alumnos. Para ello se estudiaron sus varianzas, medias, frecuencias, así como los coeficientes de regresión obtenidos sobre la legitimidad organizativa y sus dimensiones.

Se midió la legitimidad del EEES, así como la de sus dimensiones, mediante encuesta personal dirigida a los alumnos y profesores, utilizando una escala de medición tipo Likert de cinco puntos. La creación de este indicador se realizó atendiendo a la definición de legitimidad propuesta por Suchman (1995: 574), semejante a la propuesta por Thomas (2005) y posteriormente utilizado por Díez-Martín et al (2010a).

\section{Dimensiones de la legitimidad}

La revisión de la literatura sugiere la existencia de múltiples tipologías respecto a las dimensiones de la legitimidad. Stryker (1994) construye la suya a partir de las distintas conductas de comportamiento hacia las normas sociales: consentimiento, aprobación y cognición de las normas. Aldrich y Fiol (1994: 648) distinguen entre legitimidad cognitiva y sociopolítica. Scott (1995), divide la dimensión sociopolítica propuesta por Aldrich y Fiol (1994) para llegar a tres dimensiones (reguladora, normativa y cognitiva). Suchman (1995) propone una tricotomía muy similar denominándolas: pragmática, moral y cognitiva. Posteriormente Ar- chibald (2004) equipara la legitimidad sociopolítica con la reguladora y combina la normativa con la cognitiva, creando una nueva categoría llamada legitimidad cultural. Bansal y Clelland (2004) establecen una dimensión contextual a la que denominan legitimidad medioambiental de la empresa.

Al igual que Deephouse y Suchman (2008), en esta investigación se considera como más acertada la terminología propuesta por Suchman (1995). Por sí mismas, cada dimensión de legitimidad (pragmático, moral y cognitivo) supone una percepción generalizada de que las actividades organizativas son deseables, apropiadas y correctas dentro de un sistema social de normas, valores, creencias y definiciones. Sin embargo, cada una de estas dimensiones reside en distintas dinámicas de comportamiento (Díez-Martín et al., 2010b).

La legitimidad pragmática descansa sobre los intereses del entorno específico de la organización (Suchman, 1995). Una organización muestra legitimidad pragmática cuando sus objetivos se encuentran en consonancia con los intereses de sus grupos de presión y se reconoce en una organización cuando ejecuta acciones dirigidas a la obtención de esos objetivos.

La legitimidad moral, implica una evaluación normativa positiva de la organización y de sus actividades (Parsons, 1960; Aldrich y Fiol, 1994). A diferencia de la anterior, la legitimidad moral no descansa en juicios sobre si un determinado tipo de acción beneficia al evaluador, sino más bien, sobre si un determinado tipo de acción es lo que se debe hacer: una organización muestra legitimidad moral cuan- 
do sus grupos de interés consideran que los objetivos y las acciones que desarrolla para alcanzarlos son los deseables, con independencia de su utilidad para alcanzar beneficios particulares.

Respecto a la legitimidad cognitiva, ésta se corresponde con la percepción de que el desarrollo de las acciones de una organización es el más apropiado; cuando sus grupos de interés consideran que ejecuta sus estrategias de la mejor forma posible, con el mejor sistema, procedimiento, recursos, principalmente (Thomas, 2005). A diferencia de la legitimidad moral, la cognitiva se analiza evaluando la adecuación de las técnicas y los procedimientos utilizados para alcanzar los objetivos.

En este punto cabría preguntarse ¿cuál es la dimensión que ejerce una fuerza mayor sobre la legitimidad organizativa? ¿Las organizaciones que fundamentan su legitimidad, en la dimensión pragmática, poseen más legitimidad que aquellas fundamentadas en la moral o en la cognitiva? En el caso en que, para un sector de actividad, las organizaciones con mayor legitimidad pragmática sean las que poseen mayor legitimidad, lo recomendable sería desarrollar acciones dirigidas a ganar legitimidad pragmática. De igual modo ocurriría con el resto de dimensiones.

Conocer esta característica del sector permitiría a las organizaciones utilizar estrategias dirigidas a mejorar la gestión de la legitimidad, centrando sus esfuerzos en la dimensión más favorecedora en su sector. Hasta el momento, no se han encontrado estudios que demuestren que una dimensión proporcione mayor legitimidad organizativa que otra. En esta línea, Suchman (1995) sugiere que sería recomendable conocer las diferencias que se producen entre las dimensiones de la legitimidad para que las empresas puedan dirigir sus esfuerzos sobre aquellas dimensiones en las que tengan más carencias o sean más influyentes en su ámbito de actuación. Investigaciones anteriores han tratado este tema otorgando el mismo grado de importancia a todas las dimensiones de la legitimidad (DíezMartín, 2010b).

\section{Fuentes de legitimidad}

Un tema central para cualquier investigación sobre legitimidad consiste en identificar quién tiene la autoridad sobre la legitimación. Meyer y Scott (1983: 201) consideran que son fuentes de legitimidad "aquellos que tienen la capacidad de movilizar y hacer frente a las organizaciones", no tanto en términos de poder, sino en términos culturales. Dornbush y Scott (1975) señalan que las fuentes de legitimidad no están restringidas a un grupo concreto de personas. Para estos autores, las fuentes son aquellos grupos que otorgan legitimidad a las organizaciones, cuando creen que las actividades de la organización se ajustan a las políticas y normas socialmente aceptadas. Para Ruef y Scott (1998: 880) "Las fuentes de legitimidad son los grupos internos y externos, de las organizaciones, que observan y evalúan su legitimidad".

Identificar correctamente cuáles son esas fuentes, en una organización o en un sector de actividad, es fundamental desarrollar acciones dirigidas a favorecer la aceptación de su actividad y así mejorar su nivel. En esta línea, Suchman 
Análisis de las relaciones entre la legitimidad organizativa, sus fuentes y dimensiones Cruz-Suárez, A.; Díez-Martín, F.; Blanco-González, A. y Prado-Román, C.

(1995) considera necesario investigar la relación entre el entorno (los grupos de interés) y la legitimidad de las organizaciones.

Además, podría contemplarse la posibilidad que un tipo de fuente pudiese otorgar mayor legitimidad a una organización en comparación con otra fuente. Por ejemplo, los empleados de una organización podrían conferirle mayor legitimidad que los propios clientes. En este caso, la organización pondría más esfuerzo sobre el grupo de los empleados con el objetivo de ganar más legitimidad. Autores como Zimmerman y Zeitz (2002) sugieren que existe una necesidad de mayor investigación sobre la manera en que confieren legitimidad las diferentes fuentes.

\section{Relación entre la legitimidad organizativa y sus dimensiones}

Previo al análisis entre la legitimidad organizativa y sus dimensiones, se llevó a cabo un análisis ANOVA para comprobar la existencia de diferencias en las opiniones generadas por los alumnos y profesores. Los resultados de este aná- lisis indicaron que tan sólo una de las cuatro variables analizadas no era significativa $(0,05)$, mientras que las tres variables "dimensiones de la legitimidad" sí lo son, al contrario de la variable "legitimidad del EEES". A tenor de estos resultados, como se muestra en las siguientes tablas, se puede concluir que los grupos de la comunidad universitaria, de las universidades públicas de la CAM, muestran diferencias a la hora de evaluar la legitimidad del EEES, y por lo tanto es necesario analizar las respuestas de profesores y alumnos de forma independiente. El análisis de la relación entre la legitimidad y sus dimensiones desde la perspectiva de los profesores lo denominaremos modelo 1 , mientras que el análisis desde la perspectiva de los alumnos se denominará modelo 2.

La Tabla 1 muestra la media, desviación estándar y correlaciones de las relaciones entre las dimensiones de la legitimidad y la legitimidad del EEES del grupo profesores (modelo 1). La correlación entre la dimensión moral y cognitiva no ha sido significativa. En la Tabla 2 se indican los resultados del análisis de regresión entre estas variables, desde la

Tabla 1

Descriptivos y matriz de correlaciones (modelo 1)

\begin{tabular}{lccccc}
\hline & Media & Desv. típ. & $\begin{array}{c}\text { Legitimidad } \\
\text { EEES }\end{array}$ & $\begin{array}{c}\text { Legitimidad } \\
\text { Pragmática }\end{array}$ & $\begin{array}{c}\text { Legitimidad } \\
\text { Moral }\end{array}$ \\
\hline Legitimidad EEES & 2,999 & 1,158 & & & \\
Legitimidad Pragmática & 3,237 & 1,171 &, $525\left(^{* *}\right)$ & & \\
Legitimidad Moral & 3,515 & 1,256 &, $443\left(^{* *}\right)$ &, $408\left({ }^{* *}\right)$ & \\
Legitimidad Cognitiva & 1,826 & 1,058 &, $077\left(^{*}\right)$ &, $154\left(^{* *}\right)$ & $-0,03$ \\
\hline${ }^{* *}$ La correlación es significativa al nivel 0,01 & & & \\
$*$ *a correlación es significante al nivel 0,05 & & & & \\
Fuente: Elaboración propia.
\end{tabular}


Tabla 2

Regresión (modelo 1)

\begin{tabular}{lccc}
\hline & Beta & $\mathrm{t}$ & Sig. \\
\hline Legitimidad Pragmática & 0,415 & 12,851 & 0 \\
Legitimidad Moral & 0,278 & 8,601 & 0 \\
R cuadrado corregida & 0,342 & & \\
Tamaño de la muestra & 754 & & \\
\hline
\end{tabular}

Fuente: Elaboración propia.

percepción de los profesores. El análisis de regresión del grupo profesores explica el 34,2 por ciento de la varianza de la legitimidad del EEES. La dimensión cognitiva de la legitimidad del EEES no es significativa para esta fuente de legitimidad, por lo que se ha eliminado del modelo. Los coeficientes beta de las dimensiones pragmática y moral son positivos.

La Tabla 3 muestra la media, desviación estándar y correlaciones de las relaciones entre las dimensiones de la legitimidad y la legitimidad del EEES del grupo alumnos (modelo 2). La correlación entre la dimensión moral y cognitiva no ha sido significativa. En la Tabla 4 se indican los resultados del análisis de regresión entre estas variables, desde la percepción de los alumnos. El análisis de regre- sión del grupo alumnos explica el 38,5 por ciento de la varianza de la legitimidad del EEES. Los coeficientes beta de las dimensiones pragmática, moral y cognitiva son positivos.

Los resultados alcanzados, en relación a las diferencias que se producen entre las dimensiones de la legitimidad y la legitimidad organizativa, sugieren que la legitimidad del EEES depende significativa y positivamente de las dimensiones: pragmática, moral y cognitiva. Las tres dimensiones influyen positivamente sobre la legitimidad organizativa. Sin embargo el grado de influencia es dispar. Este dependería del tipo y la fuente de legitimidad.

Por un lado, atendiendo a las dimensiones de legitimidad, la dimensión pragmática es la que ejerce una mayor in-

Tabla 3

Descriptivos y matriz de correlaciones (modelo 2)

\begin{tabular}{lccccc}
\hline & Media & Desv. típ. & $\begin{array}{c}\text { Legitimidad } \\
\text { EEES }\end{array}$ & $\begin{array}{c}\text { Legitimidad } \\
\text { Pragmática }\end{array}$ & $\begin{array}{c}\text { Legitimidad } \\
\text { Moral }\end{array}$ \\
\hline Legitimidad EEES & 2,961 & 1,16116 & & & \\
Legitimidad Pragmática & 3,1065 & 1,15405 &, $598\left(^{* *}\right)$ & & \\
Legitimidad Moral & 3,3877 & 1,18306 &, $433\left(^{* *}\right)$ &, $469\left(^{* *}\right)$ & \\
Legitimidad Cognitiva & 1,9692 & 1,09525 &, $133\left(^{* *}\right)$ &, $074\left(^{*}\right)$ & 0,016 \\
\hline **La correlación es significativa al nivel 0,01 & & & \\
*La correlación es significante al nivel 0,05 & & & & \\
Fuente: Elaboración propia. &
\end{tabular}


Análisis de las relaciones entre la legitimidad organizativa, sus fuentes y dimensiones Cruz-Suárez, A.; Díez-Martín, F.; Blanco-González, A. y Prado-Román, C.

Tabla 4

Regresión (modelo 2)

\begin{tabular}{lccc}
\hline & Beta & $\mathrm{t}$ & Sig. \\
\hline Legitimidad Pragmática & 0,494 & 15,22 & 0 \\
Legitimidad Moral & 0,191 & 5,919 & 0 \\
Legitimidad Cognitiva & 0,097 & 3,4 & 0,001 \\
R cuadrado corregida & 0,385 & & \\
Tamaño de la muestra & 762 & & \\
\hline
\end{tabular}

Fuente: Elaboración propia.

fluencia sobre la legitimidad organizativa, en este ámbito de aplicación. Las evaluaciones de las fuentes de legitimidad dan más valor a la ejecución de los objetivos del EEES que a la moralidad de los propios objetivos o incluso a la forma en que éstos se aplican. Estos resultados son congruentes con la propuesta de Suchman (1995: 587), quien considera que la mejor manera de obtener legitimidad es simplemente ajustarse y cumplir con lo que quiere el entorno.

Por otro lado, la dimensión moral también ejerce un efecto positivo y significativo sobre la legitimidad del EEES, aunque este es bastante menor que el ejercido por la legitimidad pragmática. El valor de las evaluaciones morales influye, sobre la legitimidad EEES, aproximadamente la mitad que las evaluaciones pragmáticas. Respecto a la dimensión cognitiva, esta ejerce un grado de influencia muy pequeño sobre la legitimidad del EEES. Incluso para alguna de las fuentes de legitimidad, la influencia sobre la legitimidad del EEES no llega a ser significativa.

En este sentido, parece que la comunidad universitaria considera más importante que se cumplan e implanten los objetivos, sin importar los métodos o procedimientos utilizados para su ejecución. Esto puede venir motivado porque las evaluaciones de legitimidad se han realizado únicamente sobre universidades públicas, cuyo objetivo no es tanto los beneficios económicos sino la satisfacción de la comunidad. Quizás también pueda ser debido a que la comunidad considera que el sistema burocrático de las universidades públicas es el que debe aplicarse.

\section{Relación entre la legitimidad y sus fuentes}

EI análisis ANOVA entre profesores y alumnos de las universidades de la CAM, respecto a las variables legitimidad del EEES y sus dimensiones, señala que tanto para la legitimidad pragmática, moral y cognitiva, existen diferencias significativas entre las opiniones formuladas por las fuentes de legitimidad. Estos resultados reflejan que las evaluaciones de la comunidad universitaria son dispares entre sí y no pueden agregarse. Esta circunstancia ha sido ampliamente observada en la literatura (Deephouse y Suchman, 2008). Es decir, para este sector, se hace necesario medir la legitimidad organizativa atendiendo a la fuente de legitimidad. Estos resultados eran esperados teniendo en cuenta la disparidad 
existente entre los grupos de la comunidad universitaria, respecto a la formación, cultura, diferencia de edad, entre otros.

La Tabla 5 muestra las medias y la distribución de frecuencias de la legitimidad del EEES y sus dimensiones, atendiendo a las fuentes de legitimidad. Las medias obtenidas por la legitimidad del EEES y sus dimensiones son siempre mayores en el grupo de profesores que en el de alumnos, exceptuando la media de la legitimidad cognitiva, donde el grupo de alumnos evalúan esta dimensión con una puntuación un poco mayor. Las puntuaciones más repetidas coinciden, para todas las variables, en ambos grupos. Así, la moda que presenta la legitimidad del EEES es de 3 puntos sobre 5, en ambos grupos, la legitimidad pragmática consigue una puntuación de 3 puntos, la moral también obtiene 4 puntos, y la cognitiva alcanza 1 punto.

En relación a la legitimidad del EEES el $34,228 \%$ de los alumnos consideran que la legitimidad del EEES es poca o muy poca. Por el contrario, tan sólo el $28,91 \%$ de los profesores evalúan de igual manera este aspecto. La mayor diferencia se encuentra en que casi el $40 \%$ de las evaluaciones de los profesores se sitúan sobre los 3 puntos, a diferencia de los alumnos que no superan el $31,290 \%$.

Respecto a la legitimidad pragmática, el $15,243 \%$ de los profesores otorgan una evaluación de 5 puntos frente al $10,856 \%$ de los alumnos. También existe una distribución de frecuencias dispar en relación a la legitimidad moral, donde el $26,281 \%$ de los profesores la evalúan con 5 puntos frente al $19,029 \%$ de los alum- nos. Sin embargo, esta tendencia cambia cuando se observan las evaluaciones de la legitimidad cognitiva. Más de la mitad de los profesores $(51,643 \%)$ evalúan con 1 punto esta dimensión de la legitimidad, frente al $(44,572 \%)$ de los alumnos.

En la Tabla 6 se analiza el efecto de las dimensiones de legitimidad, atendiendo a las fuentes de legitimidad, sobre la legitimidad del EEES. Se observa que las dimensiones de la legitimidad, atendiendo a las evaluaciones de profesores y alumnos, explican un porcentaje similar de la varianza de la legitimidad del EEES. No obstante, el ajuste realizado por el grupo de alumnos $(0,385)$ es un poco mejor que el del grupo de profesores $(0,342)$. El efecto que tiene la legitimidad pragmática sobre la legitimidad del EEES es mayor en el grupo de alumnos que en el de profesores. Por el contrario, la magnitud del efecto de la legitimidad moral sobre la legitimidad del EEES es mayor en el grupo de profesores que en el de alumnos. El efecto de la legitimidad cognitiva sobre la legitimidad del EEES no es significativo en el grupo de profesores pero sí en el grupo de alumnos, aunque en este último caso, el efecto sobre la legitimidad del EEES es muy pequeño.

Para los alumnos, el efecto de la legitimidad pragmática sobre la legitimidad del EEES es mayor que para los profesores. Al contrario de lo que ocurre sobre el efecto de la legitimidad moral. Una posible explicación consiste en que los alumnos tienen una visión más cortoplacista de la educación superior que los profesores, y para ellos sea más importante la ejecución de los objetivos que su deseabilidad, pues en cuatro o cinco años dejarán la educación superior. 
Análisis de las relaciones entre la legitimidad organizativa, sus fuentes y dimensiones Cruz-Suárez, A.; Díez-Martín, F.; Blanco-González, A. y Prado-Román, C.

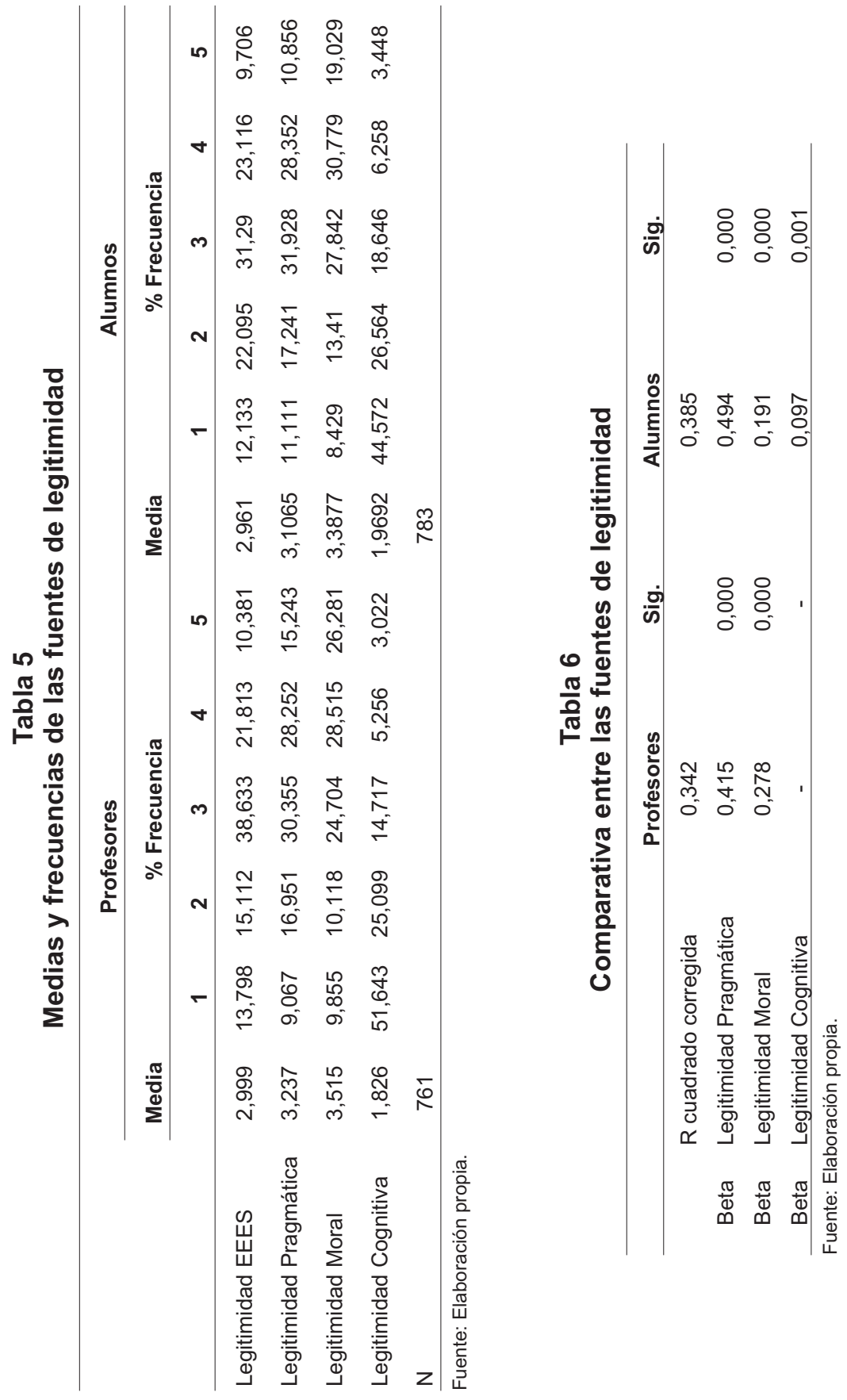


En síntesis, se ha podido confirmar empíricamente que la legitimidad depende significativa y positivamente de las dimensiones pragmática, moral y cognitiva, propuestas por Suchman (1995). Se ha demostrado que el grado de influencia de cada dimensión es dispar, pudiendo depender del tipo y fuente de legitimidad que se considere.

De este modo, se apunta que los gobiernos y las universidades podrían centrar sus esfuerzos, para conseguir una mayor aceptación del EEES, con acciones dirigidas a ganar legitimidad pragmática. Las propuestas de Suchman (1995), podrían servir para planificar una estrategia de estas características. Los beneficios de desarrollar acciones estrategias para ganar legitimidad han sido demostrados por autores como Elsbach y Sutton (1992), Alcantara et al. (2006) y Tornikoski y Newbert (2007). Las organizaciones no son simplemente elementos pasivos en el proceso de legitimación, sino que pueden trabajar activamente para influenciar y manipular las percepciones de su entorno (Oliver, 1991).

En este sentido, Suchman (1995) considera que las acciones que sirven para obtener legitimidad pragmática pueden necesitar un menor esfuerzo y, por lo tanto, serían más fáciles de ejecutar. Sin embargo, también establece que las acciones que sirven para la adquisición de legitimidad moral serían menos manipulables y más duraderas en el tiempo, aunque requerirían un mayor esfuerzo para su ejecución. De este modo, si lo importante para el EEES consiste en obtener un mayor grado de legitimidad, de forma rápida, lo más recomendable sería desarrollar acciones para conseguir legitimi- dad pragmática. Sin embargo, si el objetivo consiste en la obtención de legitimidad, de forma sostenida en el tiempo, sería más recomendable emplear los esfuerzos de la organización en desarrollar acciones para ganar legitimidad moral.

\section{Conclusiones}

La legitimidad organizativa se ha convertido en un factor clave a tener en cuenta por las empresas. Su posesión demuestra sintonía con el entorno, pues implica una percepción positiva de la actividad de la organización por parte de la sociedad. Así, para gestionar estratégicamente la legitimidad de las organizaciones se hace imprescindible conocer cómo funciona y de dónde procede. En este trabajo se analizan estas cuestiones. Por una parte se compara el efecto que tienen las distintas dimensiones de la legitimidad sobre la legitimidad global de una organización. Por otra parte, se analiza la influencia que tienen las distintas fuentes de legitimidad sobre ésta.

No todas las dimensiones de legitimidad tienen la misma influencia sobre la legitimidad global de una organización. El estudio ha señalado que, en el ámbito de la educación superior, la dimensión pragmática, a diferencia de la moral y cognitiva, es la que ejerce una mayor influencia sobre la legitimidad organizativa. Si bien estos resultados no son aplicables a todos los sectores, sugieren que a la hora de gestionar la legitimidad de una entidad, debe tenerse en cuenta el sector de actividad.

En unos sectores de actividad la legitimidad pragmática sería más importante que la cognitiva, mientras que en otros 
Análisis de las relaciones entre la legitimidad organizativa, sus fuentes y dimensiones Cruz-Suárez, A.; Díez-Martín, F.; Blanco-González, A. y Prado-Román, C.

sectores de actividad la legitimidad con mayor influencia sobre la legitimidad global podría ser la moral. De este modo, las empresas deberían desarrollar acciones estratégicas encaminadas a favorecer la obtención de aquella dimensión de legitimidad más beneficiosa en su sector. Las organizaciones no serían simplemente elementos pasivos en el proceso de legitimación, sino que podrían trabajar activamente para influenciar y manipular las percepciones de su entorno.

Pero la gestión de la legitimidad no sólo debería tener en cuenta cuál es la dimensión de la legitimidad más influyente en el sector, sino también debería discriminar entre las evaluaciones de las distintas fuentes de legitimidad. En este estudio se demuestra que no siempre es factible agregar las evaluaciones de distintas fuentes de legitimidad, porque su forma de evaluar las actuaciones de una organización podría atender a percepciones diferentes de la realidad. Por lo que sería recomendable desarrollar estrategias específicas para cada una de las fuentes de legitimidad de la empresa.

\section{Referencias Bibliográficas}

Alcantara, Lailani; Mitsuhashi, Hitoshi y Hoshino, Yasuo (2006). Legitimacy in international joint ventures: It is still needed. Journal of International Management, Vol. 12, No. 4, pp. 389-407.

Aldrich, Howard E. y Fiol, C. Marlene (1994). Fools rush in? The institutional context of industry creation. Academy of Management Review, Vol. 19, No. 4, pp. 645-670.

Archibald, Matthew E. (2004). Between isomorphism and market partitioning: How organizational competencies and resources foster cultural and sociopolitical legitimacy, and promote organizational survival en $\mathrm{C}$. Johnson (Ed.), Research in the Sociology of Organizations, Vol. 22, pp. 171-211. Amsterdam: Elsevier JAI.

Bansal, Pratima y Clelland, lain (2004). Talking trash: Legitimacy, impression management, and unsystematic risk in the context of the natural environment. Academy of Management Journal, Vol. 47, No. 1, pp. 93-103.

Barreto, llídio y Baden-Fuller, Charles (2006). To conform or to perform? Mimetic behaviour, legitimacy-based groups and performance consequences. Journal of Management Studies, Vol. 43, No. 7, pp. 1559-1581.

Baum, Joel A. C. y Oliver, Christine (1991). Institutional linkages and organizational mortality, Administrative Science Quarterly, Vol. 36, No. 2, pp. 187-219.

Brown, Andrew D. (1998). Narrative, politics and legitimacy in an IT implementation. Journal of Management Studies, Vol. 35, No. 1, pp. 35-58.

Cruz-Suárez, Ana; Prado-Román, Camilo; DíezMartín, Francisco (2014). Por qué se institucionalizan las organizaciones, Revista Europea de Dirección y Economía de la Empresa, Vol. 23, No. 1, pp. 22-30.

Deephouse, David L. (1996). Does isomorphism legitimate? Academy of Management Journal, Vol. 39, No. 4, pp. 1024-1039.

Deephouse, David L. y Suchman, Mark C. (2008). Legitimacy in Organizational Institutionalism, en Greenwood, Royston, Oliver, Christine, Suddaby, Roy y Sahlin-Andersson, Kerstin. (Eds), The Sage Handbook of Organizational Institutionalism. London: Sage.

Díez-Martín, Francisco; Blanco-González, Alicia y Prado-Román, Camilo (2010a). 
Medición de la legitimidad organizativa. El caso de las sociedades de garantía recíproca. Cuadernos de Economía y Dirección de Empresas, Vol. 43, No. 2, pp. 115-143.

Díez-Martín, Francisco; Blanco-González, Alicia y Prado-Román, Camilo (2010b). Legitimidad como factor clave del éxito organizativo. Investigaciones Europeas de Dirección y Economía de la Empresa, Vol. 16, No. 3, pp. 127143.

Díez-Martín, Francisco; Prado-Román, Camilo; Blanco-González, Alicia (2013). Beyond legitimacy: legitimacy types and organizational success, Management Decision, Vol.51, No.10. pp. 1954-1969.

DiMaggio, Paul J. y Powell, Walter W. (1983). The iron cage revisited: Institutional isomorphism and collective rationality in organizational fields, American Sociological Review, Vol. 48, No. 2, pp. 147-160.

Dornbush, Sanford M. y Scott, W. Richard (1975). Evaluation and the Exercise of Authority. San Francisco: JosseyBass.

Elsbach, Kimberly D. y Sutton, Robert I. (1992). Acquiring organizational legitimacy through illegitimate actions: A marriage of institutional and impression management theories. Academy of Management Journal, Vol. 35, No. 4, pp. 699-738.

Greenwood, Royston; Suddaby, Roy y Hinings, C. R. 2002. Theorizing change: The role of professional associations in the transformation of institutionalized fields. Academy of Management Journal, Vol. 45, No. 1,pp. 58-80.

Johnson, Cathryn (2004). Introduction: Legitimacy processes in organizations, en Cathryn Johnson (Ed.), Research in the Sociology of Organizations,
Vol. 22, pp. 1-24. Amsterdam: Elsevier JAI.

Li, Jiatao; Yang, Jing Yu y Yue, Deborah R. (2007). Identity community, and audience: How wholly owned foreign subsidiaries gain legitimacy in China. Academy of Management Journal, Vol. 50, No. 1, pp. 175-190.

Low, Brian y Johnston, Wesley (2008). Securing and managing an organizations network legitimacy: The case of Motorola China. Industrial Marketing Management, Vol. 37, No. 7, pp. 873-879.

Meyer, John W. y Rowan, Brian (1977). Institutionalized organizations: Formal structure as myth and ceremony. American Journal of Sociology, Vol. 83, No. 2, pp. 340-363.

Meyer, John W. y Scott, W. Richard (1983). Centralization and the legitimacy problems of local government, en John W. Meyer, y W. Richard Scott (Eds.), Organizational Environments: Ritual and Rationality, pp. 199-215. Beverly Hills, CA: Sage.

Oliver, Christine (1991). Strategic responses to institutional processes. Academy of Management Review, Vol. 16, No. 1, pp. 145-179.

Parsons, Talcott (1960). Structure and process in modern societies. Glencoe, IL.: Free Press.

Ruef, Martin y Scott, W. Richard (1998). A multidimensional model of organizational legitimacy: Hospital survival in changing institutional environments, Administrative Science Quarterly, Vol. 43, No. 4, pp. 877-904.

Rutherford, Matthew W. y Buller, Paul F. (2007). Searching for the legitimacy threshold. Journal of Management Inquiry, Vol. 16, No. 1, pp. 78-92.

Scott, W. Richard (1995). Institutions and organizations, Thousand Oaks, CA.: Sage. 
Análisis de las relaciones entre la legitimidad organizativa, sus fuentes y dimensiones Cruz-Suárez, A.; Díez-Martín, F.; Blanco-González, A. y Prado-Román, C.

Senés García, Belén y Llamas Sánchez, Rocío (2004). El diálogo implícito de Simon con la teoría institucional. Revista Europea de Dirección y Economía de la Empresa, Vol. 13, No. 2, pp. 61-72

Stryker, Robin (1994). Rules, resources, and legitimacy processes: Some implications for social conflict, order and change. American Journal of Sociology, Vol. 99, No. 4, pp. 847-910.

Suchman, Mark C. (1995). Managing legitimacy: strategic and institutional approaches, Academy of Management Review, Vol. 20, No. 3, pp. 571-610.

Thomas, Tom E. (2005). Are business students buying it? A theoretical framework for measuring attitudes toward the legitimacy of environmental sustainability, Business Strategy and the Environment. Vol. 14, No. 3, pp. 186-197.
Tornikoski, Erno T. y Newbert, Scott L. (2007). Exploring the determinants of organizational emergence: A legitimacy perspective. Journal of Business Venturing. Vol. 22, No. 2, pp. 311335.

Weber, Max (1946 [1922]). Class, status, party, pp. 180-95 de Max Weber Essays in Sociology, ed. H. H. Gerth y C. Wright Mills. New York: Oxford University Press.

Zimmerman, Monica y Zeitz, Gerald J. (2002). Beyond survival: achieving new venture growth by building legitimacy, Academy of Management Review. Vol. 27, No. 3, pp. 414-31.

Zucker, Lynne G. (1987). Institutional theories of organization, Annual review of Sociology, Vol. 13, pp. 443-464. Chicago: University of Chicago Press. 